\title{
On the Difference in the Monthly Sea Level between Kushimoto and Uragami, Japan*
}

\author{
Shigeo MORIYASU**
}

\section{Introduction}

We have indicated that the Kukoshio south of Shiono-misaki and the cold water region in Enshü-nada have influences on the difference in the monthly sea level between Kushimoto and Uragami (MorIYASU, 1958). In May, 1959, the Kuroshio south of Shiono-misaki moved abruptly southward and the cold water region developed (MorIYASU, 1960). According as the movement, the difference in the monthly sea level between both stations also changed remarkably. Therefore, we think it possible to ascertain the characteristics of the difference in the monthly sea level by using new data.

The distance between both stations is not great as shown in Fig. 2 (about $15 \mathrm{~km}$ ). And so, we are apt to consider that the sea level at both stations similarly fluctuates and that the difference is almost constant. However, the difference is much increased and variable when the Kuroshio is near to Shiono-misaki. This is very interesting. We shall show kinematically the possibility that such a great difference occurs in that case. Though we have not sufficient data, we can use the surface current measured with GEK in the neighbourhood of Shiono-misaki on August 31, 1956. This consideration will contribute to the conclusion that we find the close relationship between the difference in the monthly sea level and the oceanographic conditions.

2. The feature of the difference in the monthly sea level between both stations

In the previous paper (MoRIYASU, 1958), we deduced the feature of the difference in the monthly sea level as follows:

(1) When the Kuroshio is far from the coast off Shiono-misaki and the cold water

\footnotetext{
* Received Aug. 2, 1961.

** Kobe Marine Observatory
}

region is large, the difference is small and nearly constant.

(2) When the Kuroshio is near Shionomisaki and the cold water region is not large, the difference is variable and sometimes becomes an astonishing value.

The conclusion was derived from the data in 1953 1956. As the cold water region occurred in June, 1959, the conclusion should be verified on the basis of new data. We show the difference in the monthly sea level in Fig. 1, where the sea level is referred to the datum line of each tidal station. We could

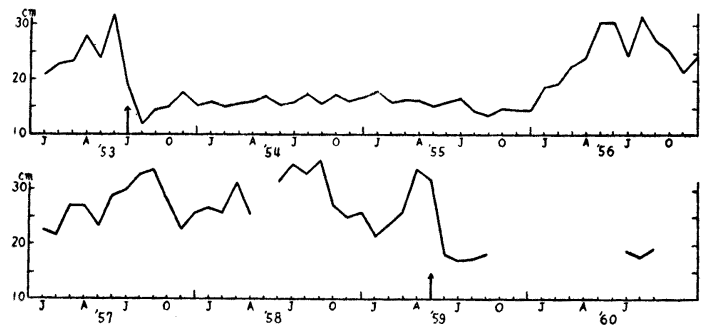

Fig. 1. The difference in the monthly sea level between Kushimoto and Uragami (Kushimoto minus Uragami). The arrow (shows the month when the current axis of the Kuroshio moved abruptly southward.

find the abrupt southward movement of the Kuroshio south of Shiono-misaki in the second half of May, 1959 and the occurrence of the cold water region in Enshū-nada (MoRIYASU, 1960), and we have the data which support that the conditions continue thereafter. On the other hand, the difference in the monthly sea level was abruptly decreased in June, 1959 and it was less by $13.7 \mathrm{~cm}$ as compared with that in the previous month, and it has been $17 \sim 19 \mathrm{~cm}$ though the data are not obtained from October, 1959 to June, 1960. The feature is in accordance with that seen in 1953 and does not contradict the deduction in the previ- 
ous paper. Accordingly, the deduction can be found to be applicable to this case. In other words, the fact mentioned above supports our conclusion derived in 1958.

\section{The relation between the difference in the monthly sea level and the current pattern}

In this section, we kinematically account for the difference in the monthly sea level as related with the current pattern.

When the Kuroshio is far from the coast south of Shiono-misaki and the cold water region is large, the southwestward current appears and the stream line is parallel to the coast and its curvature is small in the concerned area. If we consider that the geostrophic balance is nearly satisfied, the stream line nearly coincides with the line of constant elevation of the sea surface. Namely, the change of the elevation is hardly seen in the direction of motion. Though we have no data which verify the expression, it may be admitted. In this case, the difference is of nearly constant small value and its fluctuation is trivial.

Now, we discuss the second case, that is, the Kuroshio is near Shiono-misaki and the cold water region is not large. We have not sufficient data in this case, too. But, we find some available data, which were obtained on board the Shumpu Maru in the neighbourhood of Shiono-misaki on August 31, 1956. The outline of the results is shown in Fig. 2. The observations were carried out in three hours. We can consider that Station A was not situated in the Kuroshio because the surface water temperature was lower. The velocity component to at the sea surface perpendicular to the track of the vessel was obtained with GEK and so the volume transport across the track at the sea surface was computed. If we assume that the divergence vanishes in the pentagon $\mathrm{ABB}^{\prime} \mathrm{CDA}$, we can estimate the volume transport across $\mathrm{AD}$. The volume transport is obtained as follows:

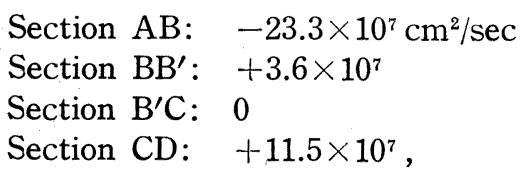

where the positive sign means the transport

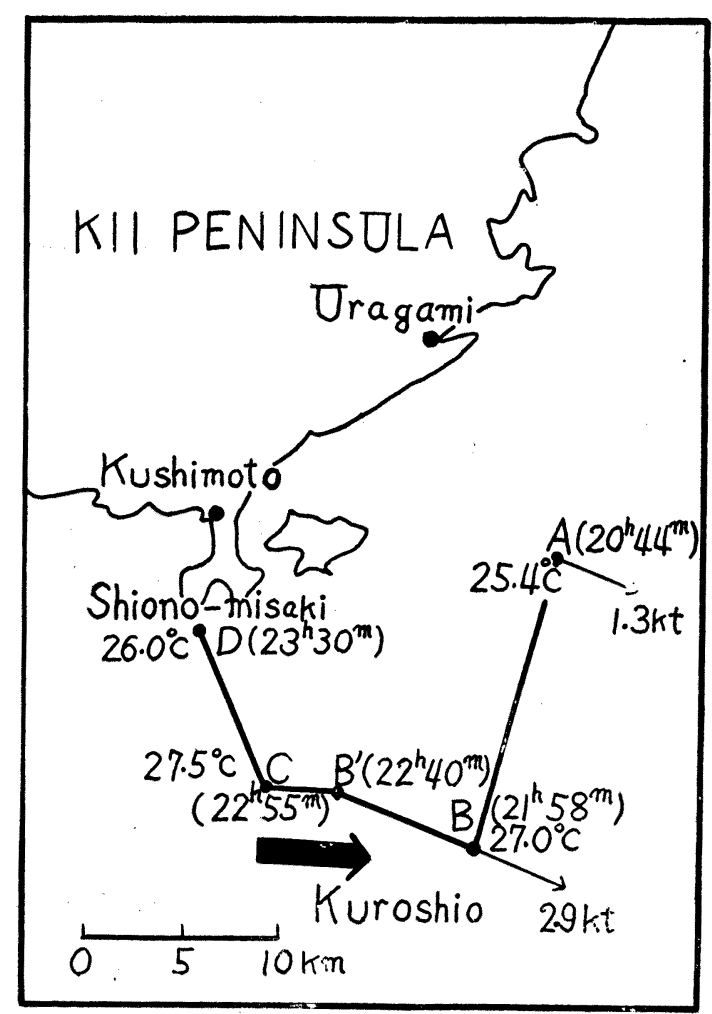

Fig. 2. The outline of the observations on August 31,1956 . We insert the time, the surface current and the surface water temperature. The track of the vessel is shown by thick line.

flowing into the pentagon. Thus, we conclude that the water flows into the pentagon across $\mathrm{AD}$ at the rate of $8.2 \times 10^{7} \mathrm{~cm}^{2} / \mathrm{sec}$. The transport must pass between Station A and Uragami. Based on these results, the current pattern in Fig. 3 is given, where the stream function is as follows:

$$
\phi=A \sin \frac{\pi}{a} x \sin \frac{\pi}{b} y .
$$

The unknown constants $A, a$ and $b$ can be decided when the current velocity at Station $A$ and the volume transport mentiond above are used. Here, the origin is taken near Kushimoto and $y$-axis is nearly along the coast line. In this case, the constants are given as follows:

$$
\begin{aligned}
A & =13.6 \times 10^{7} \mathrm{~cm}^{2} / \mathrm{sec} \\
a & =2.0 \times 10^{6} \mathrm{~cm} \\
b & =5.2 \times 10^{6} \mathrm{~cm} .
\end{aligned}
$$

If the steady state is assumed, the equation 


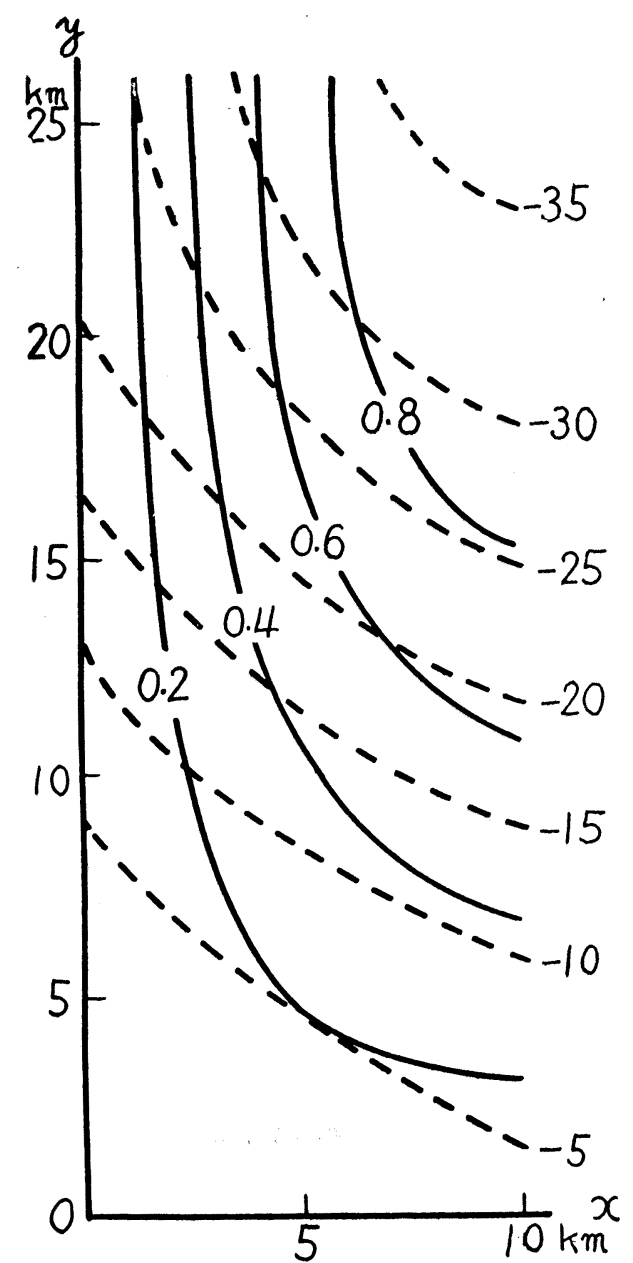

Fig. 3. The assumed current pattern and the computed elevation of the sea level in the second case. The full line is the stream line, $\phi / 13.6 \times 10^{7}$ $\mathrm{cm}^{2} / \mathrm{sec}$ and the broken line is the elevation in $\mathrm{cm}$.

of motion in the area is

$$
\begin{aligned}
& u \frac{\partial u}{\partial x}+v \frac{\partial u}{\partial y}-f v=-g \frac{\partial \zeta}{\partial x} \\
& u \frac{\partial v}{\partial x}+v \frac{\partial v}{\partial y}+f u=-g \frac{\partial \zeta}{\partial y},
\end{aligned}
$$

where $f$ is Coriolis parameter and $\zeta$ is the elevation of the sea surface. The term of lateral mixing is neglected, because $\psi$ is of order noted above and the coefficient of lateral mixing is considered to be less than $10^{8}$. Putting $u$ and $v$ derived from $\phi$ into the equation of motion and intergating the equation, we have

$$
\begin{aligned}
\zeta=\frac{1}{g}[ & \left.\left(\frac{\pi A}{2}\right)^{2}\left(\frac{1}{b^{2}} \cos \frac{2 \pi}{a} x+\frac{1}{a^{2}} \cos \frac{2 \pi}{b} y\right)\right] \\
& -f A \sin \frac{\pi}{a} x \sin \frac{\pi}{b} y \\
& -\frac{1}{g}\left(\frac{\pi A}{2}\right)^{2}\left(\frac{1}{b^{2}}+\frac{1}{a^{2}}\right) .
\end{aligned}
$$

Here, the integral constant is decided so that $\zeta$ may vanish at the origin. If $f=8 \times 10^{-5}$ $\mathrm{sec}^{-1}$ is given, we have the elevation of the sea surface shown in Fig. 3. At $x=0$ i.e. on the coast, the effect of Coriolis force vanishes and $\zeta$ is represented by

$$
\zeta=\frac{1}{g}\left(\frac{\pi A}{2 a}\right)^{2}\left(\cos \frac{2 \pi}{b} y-1\right) .
$$

The variation of $\zeta$ with $y$ on the coast is shown in Fig. 4. From Figs. 3 and 4 , we find that the great gradient can exist in such

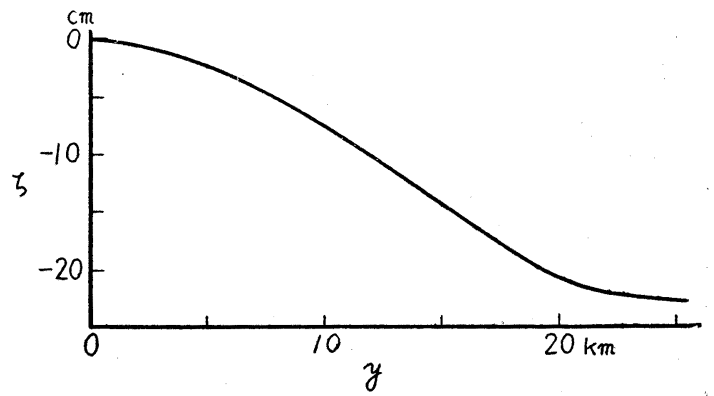

Fig. 4. The variation of the computed sea level at $x=0$ in the second case.

a case. For example, on the coast, the sea level at $y=0$ is higher by about $14 \mathrm{~cm}$ than at $y=15 \mathrm{~km}$. The greater the curvature of the stream line is, the greater the gradient of the sea level is, even if $A$ is constant. Furthermore, the difference in the sea level increases according to the increase in $A$. In the above expression, we discuss the relation between the difference in the sea level and the current pattern in the short period on the assumption that the conditions do not change during the period. The deduction mentioned above can be applied to the monthly sea level if we assume that such a current pattern appears on an average. The difference in the monthly sea level between Kushimoto and Uragami is $31.7 \mathrm{~cm}$ and $27.3 \mathrm{~cm}$ in August and September of the year, respectively. If we use the sea level referred to the standard mean sea level of Tokyo Bay (T.P.), the difference lin the monthly sea level 
is less by $13.5 \mathrm{~cm}$ than that in Fig. 1, because the datum line referred to T.P. at Kushimoto is lower by $13.5 \mathrm{~cm}$ than that at Uragami (J. M. A., 1959). Though the vertical land deformation is one factor in some cases, we have no firm basis to take account of it. This will be confirmed if the sea level is considered as related with the oceanographic conditions in the vicinity. Therefore, when we use the sea level referred to T.P., the difference in the monthly sea level between both stations is $18.2 \mathrm{~cm}$ and $13.8 \mathrm{~cm}$ in August and September of the year, respectively and has nearly the same order as computed one. As the results, a great difference in the sea level can appear when such a current pattern is found. The current pattern assumed here is not strictly ascertained to exist in the case, owing to the scantiness of the observations, but there are other data to support it (for example, SHoJi, 1958).

We classify the current pattern into two cases, but sometimes another one is found, that is, the Kuroshio flows northward on the meridian of about $136^{\circ} \mathrm{E}$ and then north eastward near the east coast of the Kii Peninsula as found in the second half of May, 1959 (MoriYasu, 1960). But, this current pattern is not stable and soon the current axis moves eastward. Accordingly, we need not consider this case. The difference in the sea level is not expected to be great in this case for the same reason as in the first case.

\section{Concluding remarks}

The difference in the monthly sea level between Kushimoto and Uragami has been discussed as related with the position of the current axis of the Kuroshio and the fluctuation of the cold water region. We have found that the results in 1959 support our conclusion derived in the previous paper. And so, our conclusion on the feature has been ascertained. The unequality of the vertical land deformation has great influence on the difference. Since the fluctuation of the difference is well corresponding to the oceanographic conditions, we think that its effect is negligible even if it exists.

We have accounted for the difference in the monthly sea level by using simple models corresponding to two cases. We are interested in the great difference and have the computed result which does not contradict the observed fact. Therefore, we can expect such great differences occur according to the current pattern given here, but unfortunately we have not sufficient data for verification of the current pattern. This is the weak point in our consideration. Though it is difficult to obtain necessary data, we hope to have observations which prove our model. As the estimation of the volume transport at the sea surface is based on the continuous record of GEK, we think that it contains considerable error. This may have influence on the computed velocity which is larger than the expected one. Furthermore, it is to be noticed that we have assumed the two dimensional motion without divergence. And we have a kinematical discussion and have not referred to the dynamical problem, that is, why the current pattern occurs in the second case. As mentioned above, there are some problems left, but we expect the discussion to contribute to revealing the influence of the oceanographic conditions upon the sea level on the coast.

\section{Acknowledgement}

The author wishes to express his hearty gratitude for his useful advices to Dr. K. Yoshida and for his discussions to Dr. K. Hishida. Furthermore, he is indebted for furnishing materials to the member of Oceanographical Section of Kobe Marine Observatory.

\section{References}

J. M. A. (1959):

Tide table for the year 1960 .

MORIYASU, S. (1958):

On the fluctuation of the Kuroshio south of Japan (4). Journ. Oceanogr. Soc. Japan. 14, 4. MORIYASU, S. (1960):

An example of the conditions at the occurrence of the cold water region. Oceanogr. Mag. 12, 2.

SHOJI, D. (1958):

On the "Shiome" at the boundary zone of the Kuroshio and the coastal water off Shiono-misaki. Rec. Oceanogr. Works, Japan. Special No. 2. (New Series) 